\title{
Perspectiva y circunstancia en la toma de decisiones: el modelo de las 4D del mundo de la empresa
}

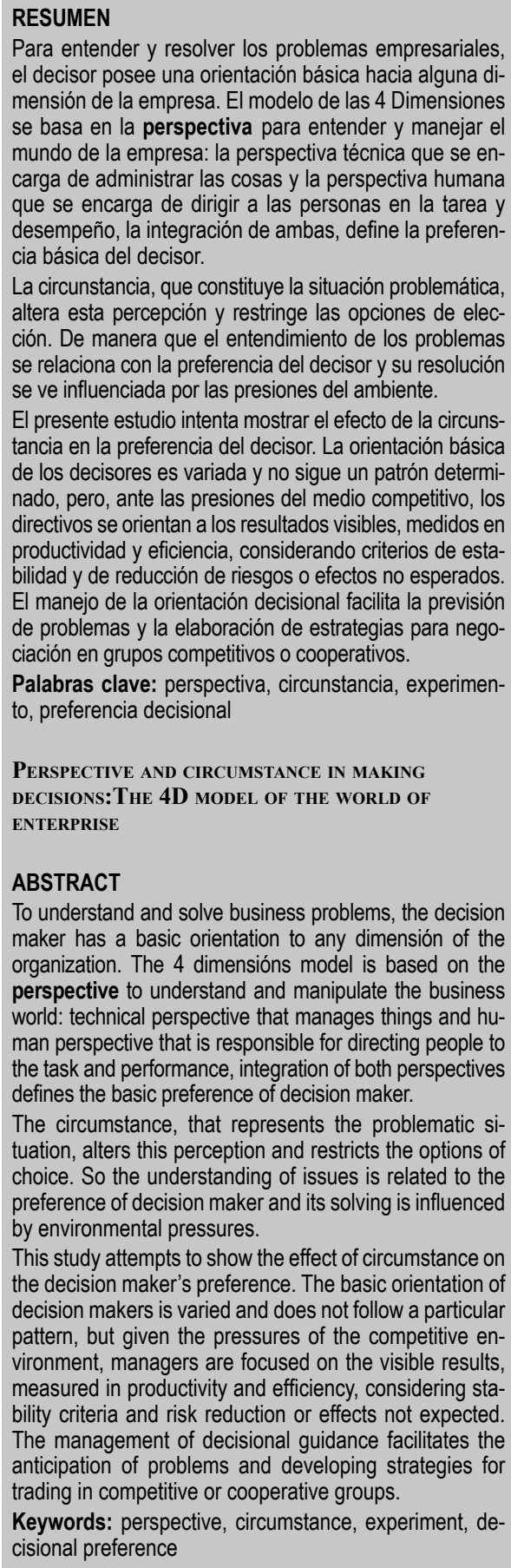

\section{ABSTRACT}

To understand and solve business problems, the decision maker has a basic orientation to any dimensión of the organization. The 4 dimensións model is based on the perspective to understand and manipulate the business world: technical perspective that manages things and human perspective that is responsible for directing people to the task and performance, integration of both perspectives defines the basic preference of decision maker.

The circumstance, that represents the problematic situation, alters this perception and restricts the options of choice. So the understanding of issues is related to the preference of decision maker and its solving is influenced by environmental pressures.

This study attempts to show the effect of circumstance on the decision maker's preference. The basic orientation of decision makers is varied and does not follow a particular pattern, but given the pressures of the competitive environment, managers are focused on the visible results, measured in productivity and efficiency, considering stability criteria and risk reduction or effects not expected. The management of decisional guidance facilitates the anticipation of problems and developing strategies for trading in competitive or cooperative groups.

Keywords: perspective, circumstance, experiment, decisional preference

\section{INTRODUCCIÓN}

El tomar decisiones para resolver problemas [16], involucra las dos dimensiónes del mundo empresarial. Por un lado la dimensión humana donde el decisor posee una perspectiva que le permite percibir los hechos y entender las situaciones sistémicas, por otro lado la dimensión del entorno que es la circunstancia o contingencia derivada de las fuerzas y elementos que presionan para la estabilidad o el cambio, y delimitan las alternativas factibles y deseables de elección [6].

El presente artículo muestra el efecto de la circunstancia ante la perspectiva del decisor, donde la cuestión básica para la toma de decisiones comprende la perspectiva que define la orientación del directivo y la circunstancia que representa las limitaciones del contexto o entorno de la organización.

\section{PERSPECTIVA Y CIRCUNSTANCIA EN LA TOMA DE DECISIONES}

La perspectiva es el weltanschauung ${ }^{2}$ del decisor, se define dentro del modelo de las cuatro dimensiónes [2] que representan la preferencia decisional, derivada de la formación del directivo, su ubicación en un plano o nivel de resolución, determina que se apliquen las soluciones según el sesgo personal del directivo, las soluciones han de ser "óptimas" en el uso de recursos (maximizadoras o minimizadora) [14] o han de ser negociadas cuando el elemento humano es preponderante.

La circunstancia es el escenario donde actúa el decisor, se define como un constructo con cuatro campos que representan los diferentes escenarios en que se asienta la situación problemática de la empresa. Contiene los elementos que limitan y constriñen las posibilidades de elección de los directivos [4]. La Figura 1 muestra los campos o escenarios donde se desenvuelve el directivo, estos

1 Magíster en Administración, Ingeniero Industrial y Economista. Profesor en la Facultad de Ingeniería Industrial, Departamento de Producción y Gestión Industrial de la UNMSM.

Email: aacevedo@speedy.com.pe.

2 Weltanschauung es un término empleado en la metodología de sistemas de Checkland pare definir la perspectiva de los actores de una situación problemática. Se define como la cosmovisión, es la imagen del entorno y del hombre que se sintetizan en los sistemas de actividad humana que brindan resultados. 
escenarios básicos se definen mediante la abstracción conceptual, la delimitación del alcance y la definición de variables, previo a fijación de magnitudes, medidas e indicadores.

\section{Estos cuatro campos son:}

- Campo I de lo dado, lo existente, la realidad, es el entorno o medio ambiente físico y de negocio en el que se desenvuelve el directivo. Desde la perspectiva no física se refiere a la operacionalización de las intuiciones puras (espacio y tiempo). Se redefinen como la posición económica y el rol social en que se ubica el directivo.

- Campo II del quehacer, es el campo de la acción humana [5] o actuación directiva orientada al empleo de los recursos recibidos para transformarlos económicamente, con beneficio económico para la sociedad [10].

- Campo III de la pertenencia y conducta dentro del grupo social, se refiere a las relaciones humanas y las normas de comportamiento para sí y para los participantes de los grupos organizacionales con los que se relaciona el directivo.

- Campo IV de la trascendencia y la proyección de los escenarios del futuro dentro de un punto focal que es el nexo causal del grupo, empresa u organización, se refiere a la percepción del cambio, devenir, desarrollo y logro histórico.

El modelo conceptual de las 4 Dimensiones del mundo de la empresa, se basa en la perspectiva que se asume para entender y para afrontar las situaciones problemáticas sistémicas [3] de las organizaciones, la perspectiva técnica y la perspectiva humana [15]. La integración de ambas perspectivas define la orientación del decisor hacia una dimensión de la empresa, la cual es delimitada por la circunstancia que restringe las opciones de elección factibles. El diseño del constructo de los campos de circunstancia es complementario al modelo conceptual 4D y se acopla para formar parte de este.

\section{INVESTIGACIÓN SOBRE EFECTO DE LA CIR- CUNSTANCIA EN LA DECISIÓN}

\section{El problema y pregunta de investigación}

La resolución de problemas dentro de las organizaciones es una de las formas que asume el trabajo directivo [8], el cual varía en función a dos grandes situaciones: $1 .^{\circ}$ ) en función al lugar en que este se encuentre dentro de la pirámide de posición y poder de la organización, $2 .^{\circ}$ ) en función a las circunstancias que rodean la situación.

La pregunta de investigación que permite verificar si existe o no influencia de la circunstancia en la elección del decisor, sería: ¿Cómo es la circunstancia que delimita y enmarca la elección de las alternativas de decisión, definidas bajo criterios y orientación del directivo?

Figura 1. Los escenarios en que actúa el decisor (campos de circunstancia)

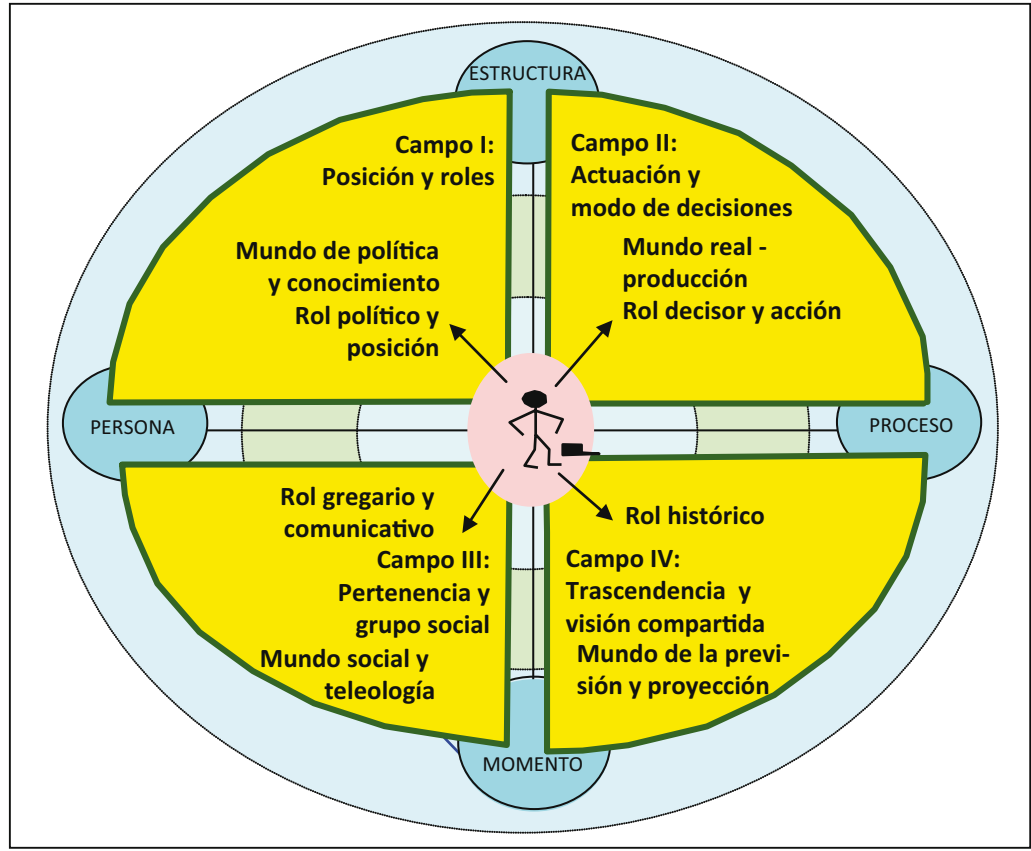




\section{La hipótesis y objetivos}

Hipótesis general: $H_{0}$

$\mathbf{H}_{0}$ : La resolución de problemas en el mundo de la empresa abarca decisiones donde la circunstancia y el contexto son irrelevantes en la elección de las alternativas.

\section{Hipótesis alterna: $\mathbf{H}_{1}$}

$\mathbf{H}_{1}$ : La resolución de problemas en el mundo de la empresa abarca decisiones secuenciales donde la elección de una alternativa es influenciada por la circunstancia y el contexto del directivo o decisor.

\section{Objetivos específicos:}

- Identificar los campos de la circunstancia que afectan la elección directiva.

- Identificar los elementos de circunstancia que influyen en la definición y resolución de problemas.

\section{Técnica de recolección de información}

El estudio cualitativo se trabajó con grupo experto y entrevista personal [4]. En el estudio cuantitativo, se eligió aleatoriamente a un grupo de directivos con grado universitario, con el criterio de ubicarse en niveles decisorios, se les solicitó el llenado de los inventarios, en diferentes momentos, se realizó en pequeños grupos.

A partir de la hipótesis, se desarrolló una matriz de consistencia que comprende las dimensiónes, los indicadores y los campos de la circunstancia, lo que se muestra en la Tabla 1. Este define la matriz básica de datos que permite la elaboración del cuestionario, y entrevista presencial, el resultado es la definición de la manera que el directivo delimita sus decisiones.

\section{Conceptos estadísticos del estudio}

- Unidad de análisis: Decisor de empresa u organización, con capacidad directiva.

- Variable: La toma de decisión (elección) por el decisor.

- Dato: Cantidad de veces que elige, según su preferencia o circunstancia.

- Instrumento de medición: Cuestionario abierto, cuestionario con escala de respuestas.

- Población: Total de directivos que toman decisiones en empresas y gobierno.

- Muestra: Grupo de decisores con nivel graduado que ejercen actividades directivas.

- Tamaño de muestra: No menos de 20 directivos

- Selección de muestra: Grupo al azar o grupo intacto.

Tabla 1. Matriz de circunstancia para la toma de decisiones y resolución de problemas

\begin{tabular}{|c|c|c|c|c|}
\hline \multirow[b]{2}{*}{$\begin{array}{l}\text { VARIABLES DE ACCIÓN } \\
\text { (EVENTO) }\end{array}$} & \multicolumn{4}{|c|}{ INDICADORES DE VARIABLE INDEPENDIENTE } \\
\hline & $\begin{array}{c}\text { FIELD } 1 \\
\mathrm{~F}_{1,4} \\
\text { Estructura-Persona }\end{array}$ & $\begin{array}{c}\text { FIELD } 2 \\
\mathrm{~F}_{1,2} \\
\text { Estructura-Proceso }\end{array}$ & $\begin{array}{c}\text { FIELD } 3 \\
F_{3,4} \\
\text { Momento-Persona }\end{array}$ & $\begin{array}{c}\text { FIELD } 4 \\
\text { F }_{3,2} \\
\text { Momento-Proceso }\end{array}$ \\
\hline $\mathrm{T}_{1}=$ Mando & Poder & Resultado & Tarea & Velocidad \\
\hline $\mathrm{T}_{2}=$ Emprendedor & Dinero & Satisfacción & Emulación & Antecedentes \\
\hline $\mathrm{T}_{3}=$ Movilidad social & Ejemplo de éxito & Trabajo & Imagen personal & Resultados \\
\hline $\mathrm{T}_{4}=$ Dilema & Control de recursos & Asignación de recursos & Efecto del recurso & Oportunidad de recursos \\
\hline $\mathrm{T}_{5}=$ Conflicto & Intereses & Eficiencia & Ética & Competencias \\
\hline $\mathrm{T}_{6}=$ Resolución de problemas & Mantener & Productividad & Satisfacción & Cambiar \\
\hline $\mathrm{T}_{7}=$ Cambio & Democracia & Mercado & Revolución & Planeamiento \\
\hline $\mathrm{T}_{8}=$ Orden & Respeto & Honradez & Beneficios & Visión compartida \\
\hline $\mathrm{T}_{9}=$ Espíritu de grupo & Bienes & Objetivos & Misiones & Visiones \\
\hline $\mathrm{T}_{10}=$ Virtudes & Justicia & Laboriosidad & Honestidad & Perseverancia \\
\hline Indicador & Posición & Actuación & Pertenencia & Trascendencia \\
\hline
\end{tabular}

Fuente: Elaboración propia. 


\section{La población del estudio}

La población comprende a los decisores de gestión de organizaciones en el contexto del Perú. Estas organizaciones se definen como empresa privada y gobierno. La determinación del tamaño del mercado (decisores) se considera un problema de magnitud, cuya cuantificación exacta implicaría un elevado costo y un insumo de tiempo significativo, por lo que se considera el método Fermi (cálculo de una serie de estimados que llevan a la respuesta correcta).

La fórmula para estimar el número de directivos decisores en empresas y organizaciones se presenta en la ecuación siguiente:

$$
\begin{aligned}
& D=\text { total de decisores de nivel estratégico*ra } \\
& D=(\text { Dep }+ \text { Dpr }+ \text { Dgc }+ \text { Dgd })^{*} r a \\
& D= \\
& \quad((D g m e+D p e+D m c)+(\text { Dmm }+ \text { Drt })+ \\
& \quad(D g c+D g d)){ }^{*} r a
\end{aligned}
$$

donde:

Dep $=$ decisores empresa privada

Dgme $=$ Decisores gran y mediana empresa $=$ fracción del total de personal

Dpe $=$ Decisores pequeña empresa $=$ fracción del total de per sonal PE

Dmc $=$ Decisores micro empresa $=$ fracción del total de personal $\mathrm{mE}$

Dpr $=$ otros decisores de empresa privada $=$ fracción del total de trabajadores

Dmm $=$ Mandos medios de empresa privada

Drt $=$ Representantes de trabajadores organizados

$\mathrm{Dgc}=$ Decisores gobierno central $=$ fracción del total de funcionarios

$\mathrm{Dgd}=$ Decisores gbno descentralizado $=$ funcionarios gbno regional y alcaldías

$\mathrm{Dpr}=$ Decisores profesionales $=$ fracción del total de PEA adec.empleada

ra $=$ ratio-ajuste $=$ PEA2012/PEA2009 pro crecimiento poblacional $=1.00$

Considerando el tamaño de la población de decisores, se ha empleado la estadística inferencial para determinar muestras aleatorias para el estudio, con el supuesto que su comportamiento tiende a una distribución normal.

Con datos estadísticos al año 2009 [11], se realizó la estimación de los decisores en el ambiente organizacional del país, que se resumen en la fórmula siguiente.

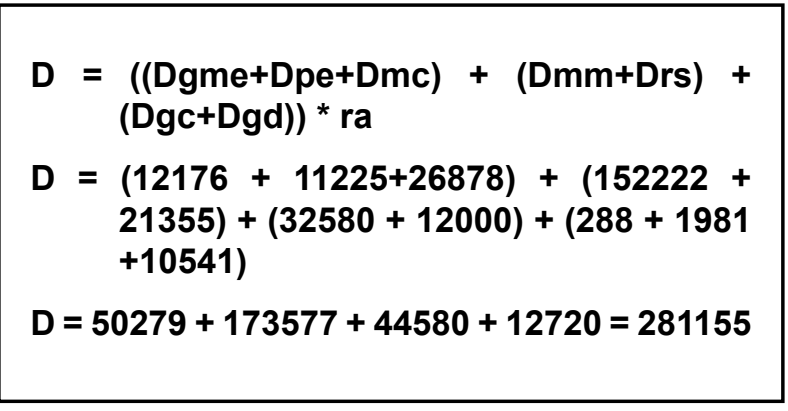

Para una población económicamente activa de 16 millones, con 6 millones adecuadamente empleados (Estimado de INEI (2009). Boletín Especial N. ${ }^{\circ}$ 20 "Perú: Estimaciones y Proyecciones de PEA Urbana y Rural, 2000- 2015"), se estima que los decisores en las organizaciones bordean los 300,000, donde el $80 \%$ corresponde a decisores en la empresa privada y el $20 \%$ restante se encuentra en el sector público (gobierno central, aparato público, gobierno descentralizado).

La Tabla 2 muestra el resultado del modelo elaborado, empleando la técnica Fermi.

\section{Diseño de la muestra}

Dada la dificultad de identificar al número de decisores por regiones se determinaron cuatro segmentos geográficos: $1 .^{\circ}$ ) Lima Metropolitana, $2 .^{\circ}$ ) Sur (Arequipa), $3 .^{\circ}$ ) Norte (Huacho), $4 .^{\circ}$ ) Oriente (Pto. Maldonado), mediante talleres académicos con profesionales (estudios de doctorado y maestría). El tamaño de muestra se determinó mediantes estadística inferencial para muestras donde no se conoce la desviación estándar y buscando un nivel de confianza de 95\%.

Se consideró veinticinco decisores (profesionales, académicos, egresados universitarios) por muestra aleatoria, el criterio común es que poseen grado académico o se encuentra en proceso.

Después del diseño de la herramienta de registro de datos, se empleó la técnica de la encuesta para la recolección de datos, la cual registra y permite medir los resultados individuales de cada variable por cada una de las dimensiónes, y la sistematización y análisis estadístico.

\section{DISEÑO EXPERIMENTAL DE LA INVESTIGA- CIÓN}

\section{El diseño del experimento}

El diseño ha sido elaborado para identificar, inicialmente si la preferencia de los decisores por una o 
Tabla 2. Estimado del número de decisores en organizaciones- Método Fermi

MERCADO OBJETIVO- RESUMEN

\begin{tabular}{|c|c|c|c|c|c|c|c|}
\hline & TARGET & Mercados & $\mathrm{N} .^{\circ}$ decisores & Totales & $\%$ & N. ${ }^{\circ}$ decisores & $\%$ \\
\hline \multirow{2}{*}{$\begin{array}{l}\text { Decisores de } \\
\text { Empresa privada }\end{array}$} & $\begin{array}{l}\text { Directivos } \\
\text { Profesionales }\end{array}$ & $\begin{array}{l}\text { A1 } \\
\text { A2 } \\
\text { A3 }\end{array}$ & $\begin{array}{l}12,176 \\
11,225 \\
26,878\end{array}$ & 50,279 & 18 & 223,856 & 80 \\
\hline & Mando Medio & $\begin{array}{l}\text { D1 } \\
\text { D2 }\end{array}$ & $\begin{array}{l}152,222 \\
21,355\end{array}$ & 173,577 & 62 & & \\
\hline \multirow{3}{*}{$\begin{array}{l}\text { Decisores de } \\
\text { gobierno }\end{array}$} & Gbno.Central & $\begin{array}{l}\mathrm{C} 1 \\
\mathrm{C} 2\end{array}$ & $\begin{array}{l}32,580 \\
12,000\end{array}$ & 44,580 & 16 & 57,300 & 20 \\
\hline & $\begin{array}{c}\text { Gbno. } \\
\text { Descentralizado }\end{array}$ & $\begin{array}{l}\text { B1 } \\
\text { B2 } \\
\text { B3 }\end{array}$ & $\begin{array}{c}288 \\
1,981 \\
10,451\end{array}$ & 12,720 & 5 & & \\
\hline & Total & $A+B+C+D$ & & 281,155 & 100 & 281,155 & 100 \\
\hline
\end{tabular}

Fuente: Elaboración propia

más dimensiónes es uniforme o dispersa, luego se introduce un elemento experimental que es la presión del contexto o circunstancia, para determinar si las decisiones iniciales se mantienen, o si el decisor modifica su elección en función a dichas presiones.

Para el segmento uno centro se ha considerado un diseño experimental Solomon [9] con tres grupos de control a fin de verificar la pertinencia del instrumento de recolección. Para los segmentos de provincias solo se considera el grupo aleatorio de tratamiento, no se ha considerado necesario el grupo de control.

Los resultados grupales se miden mediante la dispersión en un gráfico $x-y$, su ubicación xpromedioypromedio en un determinado cuadrante del modelo de campos de circunstancia, define el efecto total de las fuerzas ambientales.

Básicamente, se identifican los campos o escenarios de la circunstancia que afectan la elección directiva, los que se construyen a partir de un par de elementos que son las dimensiónes que delimitan el escenario en que actúa el decisor.

La Tabla 3 muestra el diseño experimental, donde

$$
X 1=X 1=X n ; n=5 .
$$

En la Tabla 4 se muestran los resultados para cada grupo aleatorio, antes y después de la manipulación de la variable del experimento (evento a resolver).

\section{El modelo lineal:}

Para la investigación sobre preferencias en la toma de decisiones, se emplea el modelo de regresión lineal y la prueba de hipótesis mediante análisis de varianza.

Considerando:

$\mu=$ media total

Error o residuo: variables aleatorias que se desvían de la media, independientes y normales $N\left(0, \sigma_{i}^{2}\right)$

$$
\varepsilon \mathrm{ij}=\mathrm{Xij}-\mu \mathrm{i}
$$

entonces: $X_{i j}=\mu_{i}+\varepsilon_{i j}$,

$$
\mathrm{i}=1,2,3,4, \mathrm{j}=1,2, \ldots 25
$$

\section{Efecto del tratamiento i:}

$$
\alpha \mathbf{i}=\mu \mathbf{i}-\mu
$$

donde:

$$
\sum_{i=1}^{k} \alpha_{i}=0
$$


Tabla 3. Diseño experimental y su efecto en la calidad de las decisiones

\begin{tabular}{|c|c|c|c|c|}
\hline Segmento & \multicolumn{2}{|c|}{ 1ra. parte } & \multicolumn{2}{|c|}{ 2da. parte } \\
\hline $\begin{array}{l}\text { Centro } \\
\text { (Lima Metropolitana) }\end{array}$ & $\begin{array}{l}\text { Ga1 } \\
\text { Ga2 } \\
\text { Ga3 } \\
\text { Ga4 }\end{array}$ & $\begin{array}{l}O_{1} \\
O_{3}\end{array}$ & $\begin{array}{l}x_{1} \\
x_{2}\end{array}$ & $\begin{array}{l}\mathrm{O}_{2} \\
\mathrm{O}_{4} \\
\mathrm{O}_{5} \\
\mathrm{O}_{6}\end{array}$ \\
\hline $\begin{array}{l}\text { Sur } \\
\text { (Arequipa) }\end{array}$ & Ga5 & $\mathrm{O}_{7}$ & $x_{3}$ & 08 \\
\hline $\begin{array}{l}\text { Norte } \\
\text { (Huacho) }\end{array}$ & Ga6 & $\mathrm{Og}_{9}$ & $x_{4}$ & 010 \\
\hline $\begin{array}{l}\text { Oriente } \\
\text { (Pto. Maldonado) }\end{array}$ & $\mathrm{Ga} 7$ & $\mathrm{O}_{11}$ & $\times 5$ & 012 \\
\hline $\begin{array}{l}\text { Efecto en la } \\
\text { calidad de las } \\
\text { decisiones }\end{array}$ & $\begin{array}{r}\text { Eficacia } \\
\text { decisione } \\
\text { fine }\end{array}$ & $\begin{array}{l}\text { ciencia de } \\
\text { criterios y } \\
\text { ersos }\end{array}$ & $\begin{array}{r}\text { Eficacia } \\
\text { decisione } \\
\text { propósi }\end{array}$ & $\begin{array}{l}\text { ficiencia de } \\
\text { on criterios y } \\
\text { unificados }\end{array}$ \\
\hline
\end{tabular}

Fuente: Elaboración propia

Tabla 4. Resultados del estudio experimental

\begin{tabular}{|c|c|c|c|c|c|}
\hline \multirow{2}{*}{$\begin{array}{c}\text { GRUPO } \\
\text { ALEATORIO }\end{array}$} & \multicolumn{2}{|c|}{ Coordenadas $\mathrm{x}, \mathrm{y}$} & \multicolumn{2}{|c|}{ Campo de acción decisional } & \multirow{2}{*}{$\begin{array}{c}\text { EFECTO } \\
\text { DE X }\end{array}$} \\
\hline & 1ra. parte & 2da.parte & 1ra. parte & 2da.parte & \\
\hline Ga1 & $1.0,-2.4$ & $6.1,4.9$ & IV & $\|$ & \\
\hline Ga2 & $0.8,-2.3$ & $0.5,-1.9$ & IV & IV & \\
\hline Ga3 & & $4.2,5.8$ & & II & \\
\hline Ga4 & & $1.4,0.1$ & & II-IV & \\
\hline Ga5 & $1.5,0.8$ & $4.6,4.1$ & II & II & \\
\hline Ga6 & $3.4,4.5$ & $7.8,8.0$ & ॥ & $\|$ & \\
\hline Ga7 & $0.9,0.6$ & $7.4,6.5$ & II & II & \\
\hline \multicolumn{6}{|l|}{ ajustado: } \\
\hline Ga1 & $1,-2$ & 6,5 & IV & ॥ & sí \\
\hline Ga2 & $1,-2$ & $1,-2$ & IV & IV & NO \\
\hline Ga3 & & 4,6 & & II & sí \\
\hline Ga4 & & 1,0 & & II-IV & NO \\
\hline Ga5 & 2,1 & 5,4 & II & ॥ & sí \\
\hline $\mathrm{Ga} 6$ & 3,5 & 8,8 & II & ॥ & sí \\
\hline $\mathrm{Ga} 7$ & 1,1 & 7,7 & ॥ & ॥ & sí \\
\hline ajustado: & & & & & \\
\hline
\end{tabular}

Fuente: Elaboración propia 
El modelo de un factor completamente aleatorio:

$$
x_{i j}=\mu_{i}+\alpha_{i}+\varepsilon_{i j}
$$

\section{1. ${ }^{\circ}$ Hipótesis}

$\mathrm{HO}: \mu 1=\mu 2=\mu 3=\mu 4=\mu$

Todas las $\mu \mathrm{i}$ son iguales ; donde $\mathrm{i}=1,2, . . \mathrm{k}, \mathrm{k}=4$

ó: no hay efecto de $\mathrm{k}$ tratamientos)

$\mathrm{H} 1$ : No todas las $\mu \mathrm{i}$ son iguales ; donde $\mathrm{j}=1,2$, 3,4

donde:

$\mu \mathrm{i}:$ Media de las elecciones por el tratamiento i

2. Nivel de significancia y nivel de confianza (n.s. y n.c)

Nivel de significancia $\alpha=0.05$

Nivel de confianza $1-\alpha=0.95$

\section{3. ${ }^{\circ}$ Cálculo mediante SPSS:}

Se muestra en las Tablas 5 y 6.

4. ${ }^{\circ}$ Prueba $F$ para análisis de varianza de un factor (ver Figura 2).

\section{5. ${ }^{\circ}$ Resultados de la muestra}

Para un nivel de significación de 0.05 el $\mathrm{F}$ calculado(24.08) es superior al F teórico (2.70), lo que indica que los promedios no corresponden a la misma población. Por lo tanto se rechaza la hipótesis nula que afirma que los directivos, en la elección de alternativas, no son afectados por la circunstancia.

No se puede rechazar la hipótesis alternativa que indica que las elecciones de los directivos son afectados por la circunstancia, en el estudio, su elección se ve claramente modificada. Lo que se resume en la Figura 2.

Tabla 5. Estadísticos descriptivos

Variable dependiente: PREFERENCIA

\begin{tabular}{cccccccccc}
\hline & $\begin{array}{c}\text { N. } \\
\text { Límite } \\
\text { inferior }\end{array}$ & $\begin{array}{c}\text { Media } \\
\text { Límite } \\
\text { superior }\end{array}$ & $\begin{array}{c}\text { Desviación } \\
\text { típica } \\
\text { Límite inferior }\end{array}$ & $\begin{array}{c}\text { Error típico } \\
\text { Límite } \\
\text { superior }\end{array}$ & $\begin{array}{c}\text { Intervalo de confianza para } \\
\text { la media al 95\% }\end{array}$ & $\begin{array}{c}\text { Mímimo } \\
\text { inferior }\end{array}$ & $\begin{array}{c}\text { Límite supe- } \\
\text { ínior }\end{array}$ & $\begin{array}{c}\text { Máximo } \\
\text { ímite } \\
\text { inferior }\end{array}$ & $\begin{array}{c}\text { Límite } \\
\text { superior }\end{array}$ \\
\hline 1 & 25 & 25.36 & 3.650 & .730 & 23.85 & 26.87 & 18 & 32 \\
\hline 2 & 25 & 30.16 & 4.089 & .818 & 28.47 & 31.85 & 21 & 36 \\
\hline 3 & 25 & 24.04 & 4.420 & .884 & 22.22 & 25.86 & 17 & 35 \\
\hline 4 & 25 & 20.44 & 4.184 & .837 & 18.71 & 22.17 & 14 & 31 \\
\hline Total & 100 & 25.00 & 5.339 & .534 & 23.94 & 26.06 & 14 & 36 \\
\hline
\end{tabular}

Fuente: Elaboración propia empleando SPSS

Tabla 6. Pruebas de los efectos inter-sujetos

Variable dependiente: preferencia personal

Elección personal

\begin{tabular}{cccccc}
\hline & $\begin{array}{c}\text { Suma de cua- } \\
\text { drados }\end{array}$ & gl & $\begin{array}{c}\text { Media cuadrá- } \\
\text { tica }\end{array}$ & F & Sig. \\
\hline Inter-grupos & 1211.760 & 3 & 403.920 & 24.081 & .000 \\
\hline Intra-grupos & 1610.240 & 96 & 16.773 & & \\
\hline Total & 2822.000 & 99 & & & \\
\hline
\end{tabular}

Fuente: Elaboración propia empleando SPSS 
Figura 2. Prueba de hipótesis de la investigación

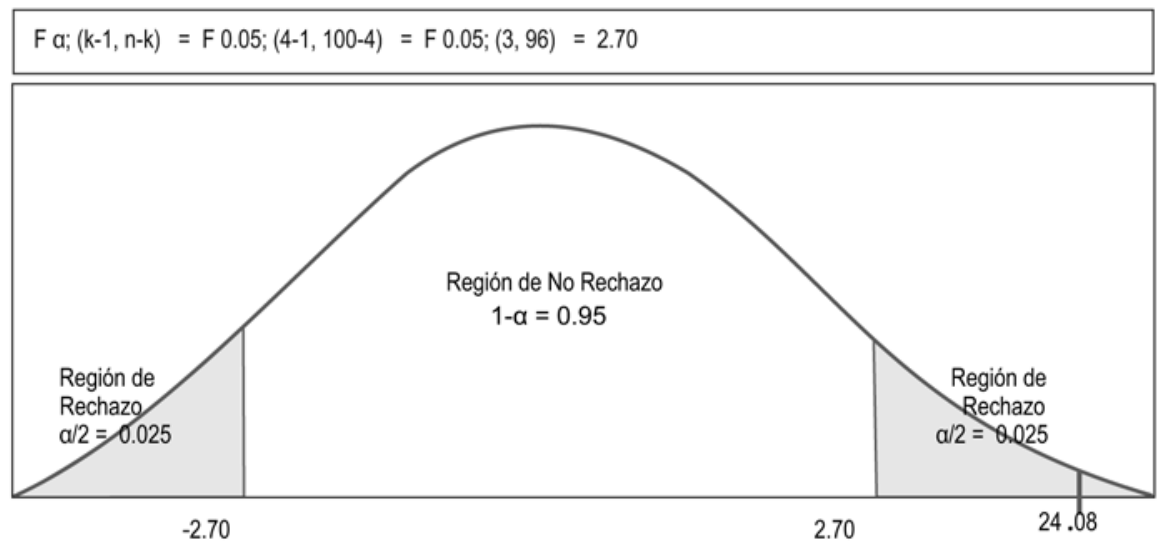

Fuente: Elaboración propia empleando SPSS

\section{RESULTADOS DEL ESTUDIO EN EL SEGMENTO CENTRO}

Los resultados permiten verificar que las decisiones considerando el efecto de la circunstancia orientan las decisiones hacia el campo II, que se define como del utilitarismo y los resultados [12]. Por el contrario, según la primera parte del estudio, si no existiere presión de la circunstancia, la preferencia sería más dispersa y menos orientada hacia un campo específico.

Detallando los hallazgos del grupo aleatorio 1 , se observa gráficamente, la manera en que se reorienta la preferencia en la muestra 1 ga1. La Figu- ra 3 muestra los resultados $\mathrm{O} 2$ que derivan de la reorientación de 01 .

Gráficamente se resume en la Figura 4. El perfil inicial (de orientación a la dimensiónes) es diferente al perfil final resultante.

$$
\mathrm{O}_{1} \text { es diferente } \mathrm{a}_{2}
$$

Lo que señala que la intervención $\mathbf{X}_{1}$ tiene efecto en la reorientación de la preferencia del decisor. En general, el perfil previo tiene menor dispersión en los promedios de preferencia por dimensión, en cambio el perfil posterior presenta mayor varia-

Figura 3. Resultados grupo 101 vs $\mathrm{O} 2$

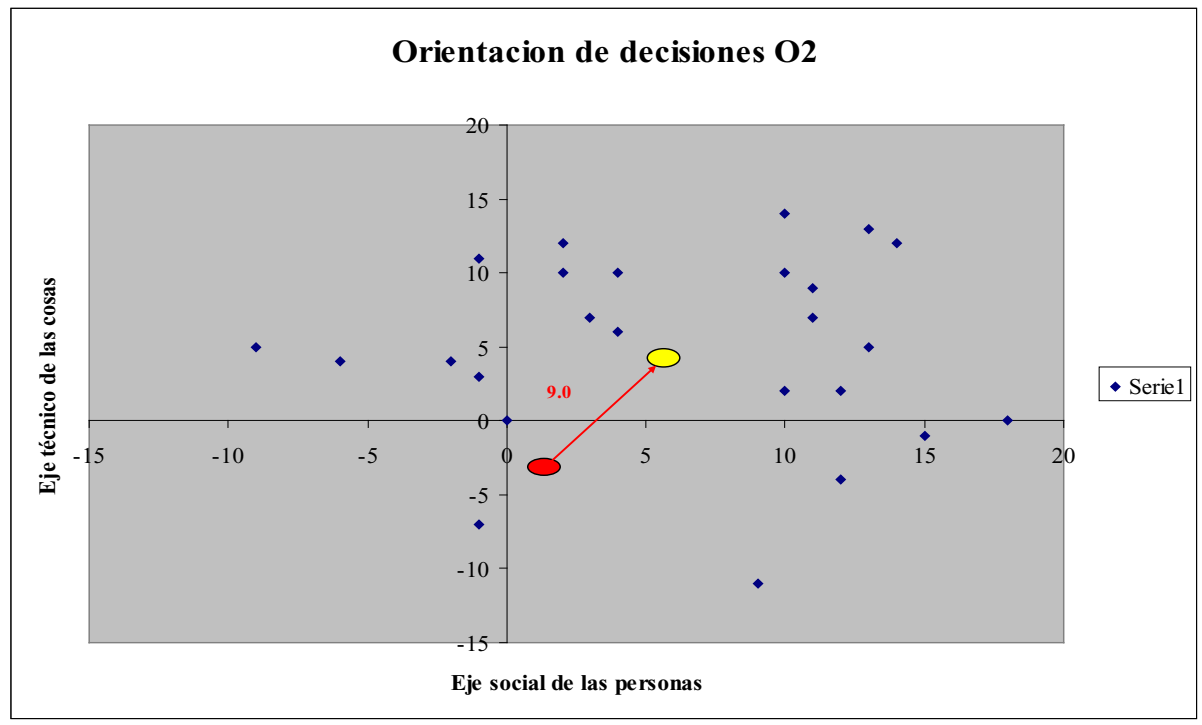

Fuente: Elaboración propia empleando SPSS y MS-Excel 
Figura 4. Efecto de la circunstancia en el perfil de preferencia $01 \neq 02$

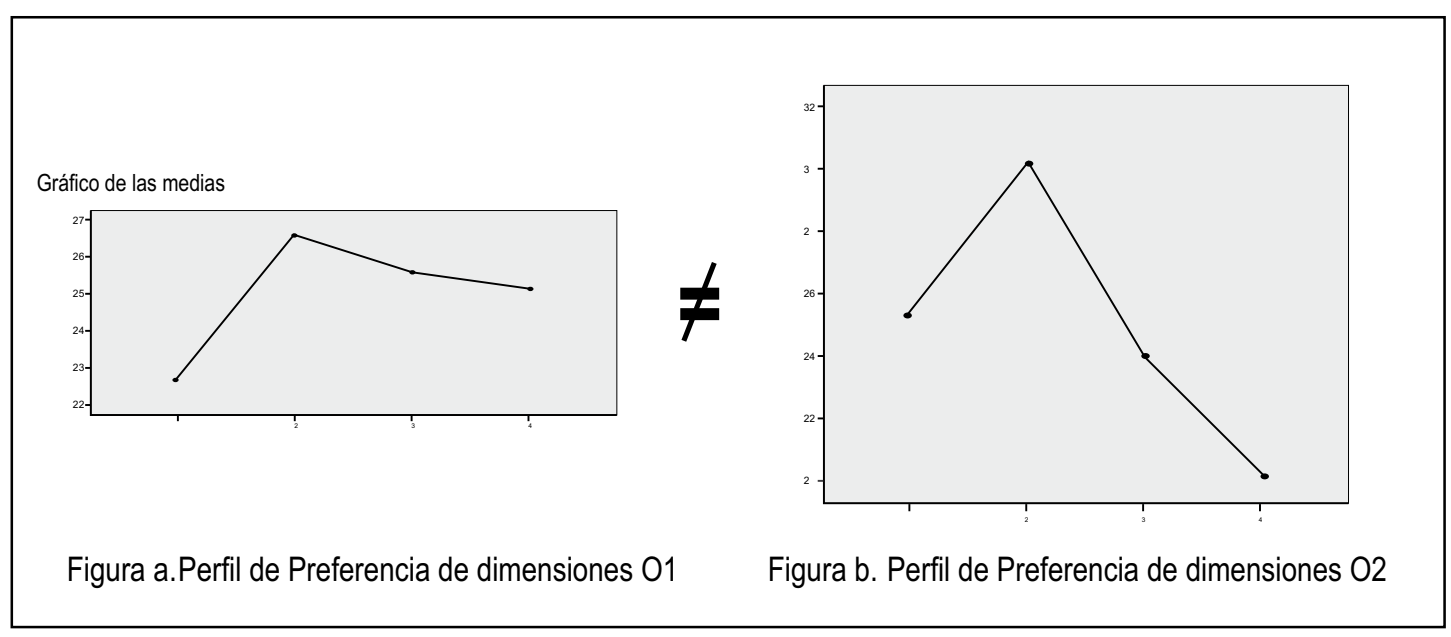

Fuente: Elaboración propia empleando SPSS y MS-Excel

ción, donde se enfatiza la dimensión 1 estructura y la dimensión 2 proceso. El entorno que constituye la circunstancia, se enfoca en la combinación de recursos para transformarlos, la preferencia hacia el campo 2 es el resultado natural de los decisores que actúan en escenarios que exigen resultados y ejercen rol y actividad productiva.

La Figura 5 muestra que no existen variaciones significativas en los resultados $\mathrm{O} 3$ y $\mathrm{O} 4$, obtenidos del estudio experimental en la muestra ga2, donde no hay intervención de la circunstancia, ni para la respuesta 3 , ni para la respuesta 4 .

Lo que señala que el perfil inicial es semejante al perfil final resultante. Cuando no existe alguna intervención circunstancial que influencie al decisor $(\mathrm{Xn})$, no se presentan cambios significativos en el perfil de preferencia del decisor.

\section{O3 es semejante a 04}

En el grupo muestral 3, las preferencias se concentran en las dimensiónes estructura y proceso (campo 2).Ante las presiones del entorno para obtener resultados, los decisores asumen posturas utilitaristas, orientando su elección hacia la eficacia, la productividad y la eficiencia, o sea, hacia las tareas que generan resultados útiles. El perfil del grupo aleatorio 4 , muestra que las preferencias del grupo son menos dispersas y se orientan hacia las dimensiónes proceso y persona, y menos a estructura y momento. En el estudio, la desviación media absoluta de las coordenadas de la respuesta $\mathrm{O} 6$ frente a la respuesta 05 , confirma que ambos resultados no son semejantes.

\section{O5 es diferente a 06}

La Figura 6 presenta un perfil final y único del grupo 3 que es semejante al perfil después de la intervención, del grupo 1. El resultado 05 del grupo 3 es semejante al resultado $\mathrm{O} 2$ del grupo aleatorio 1.

\section{5 es semejante a $\mathrm{O} 2$}

Lo que señala que la intervención X2: tiene efecto en la reorientación de la preferencia del decisor del ga3, tal como el efecto de la intervención X1 en la orientación del ga1.

\section{X2 tiene efecto en la preferencia del decisor,}

\section{tal como X1}

Ambos resultados, señalan que, cuando interviene la circunstancia, a través de las fuerzas del entorno [13], el decisor redefine o reafirma su orientación hacia las dimensiónes de la estructura (la estabilidad del ambiente y la administración de recursos) y del proceso (la búsqueda de resultados a través de la gestión de los procesos de transformación), enfocando las coordenadas en el campo 2 del utilitarismo y los resultados, enfocado en lo útil y lo que sirve, de manera que se alcancen los propósitos definidos para el ente empresarial. Por el contrario, el resultado $\mathrm{O} 6$ de la muestra aleatoria 4, en forma semejante a los resultados $\mathrm{O} 1, \mathrm{O} 3$ y $\mathrm{O} 4$, donde el decisor define su orientación personal, sin la intervención de las fuerzas del entorno, señala que las preferencias se pueden ubicar en cualquier campo, donde no se percibe sesgo claro y definido hacia alguna dimensión del mundo de la empresa. 
Figura 5. Efecto de la circunstancia en el resultados grupo 203 vs $04+$

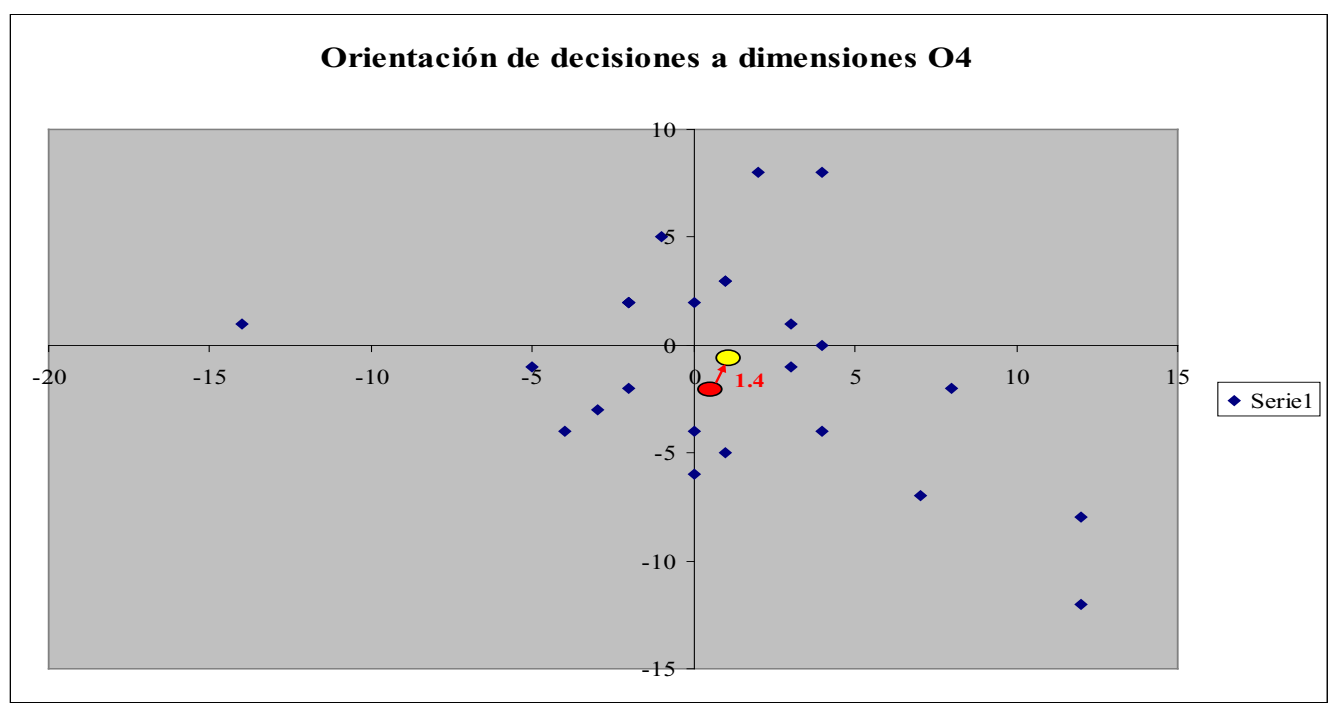

Fuente: Elaboración propia empleando SPSS y MS-Excel

Figura 6. Efecto de la circunstancia en el perfil de preferencia 05 y 02

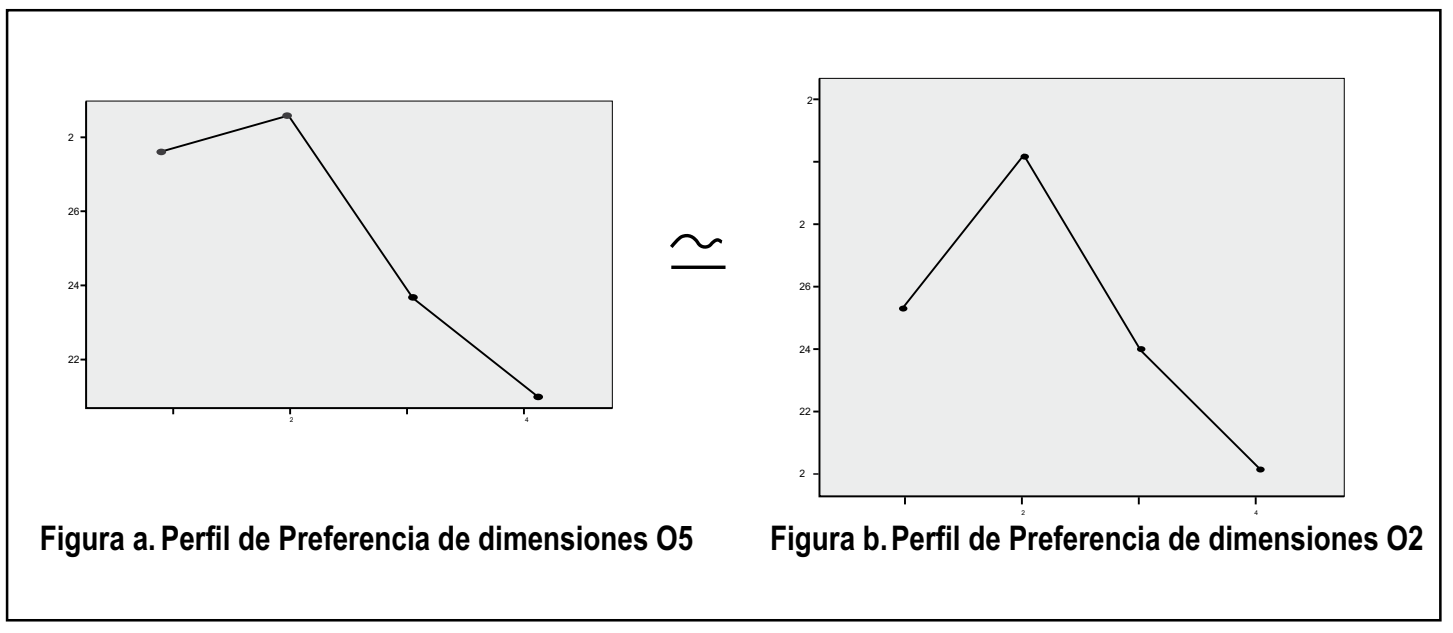

Fuente: Elaboración propia empleando SPSS y MS-Excel

Con mayor detalle, en la Figura 7 se analiza la muestra 1, donde se observa que la media de las dimensiónes $(\mathrm{x}, \mathrm{y})$ ha variado, ubicándose dentro del perfil del campo 2. Lo que no se ha determinado si estos cambios derivados de las presiones de la circunstancia, son temporales mientras dure la situación de inestabilidad, o si se tornan permanentes.

De acuerdo a los resultados, los directivos concentran la orientación de sus decisiones hacia resul- tados y la estabilidad (84\%) donde la importancia de los resultados del campo 1 se eleva hasta $64 \%$, mientras antes llegaba a $32 \%$., el campo 4 del orden y posición pasa de $12 \%$ hacia el $20 \%$.

Se observa que, en una situación problemática a resolver, la percepción de lo relevante es distorsionada por la introducción de la circunstancia, de manera que las decisiones bajo las presiones del ambiente son diferentes a las decisiones tomadas sin presiones de ningún tipo. 
Figura 7. Perfil de orientación a la decisión - Resultados 1y 2 del ga1

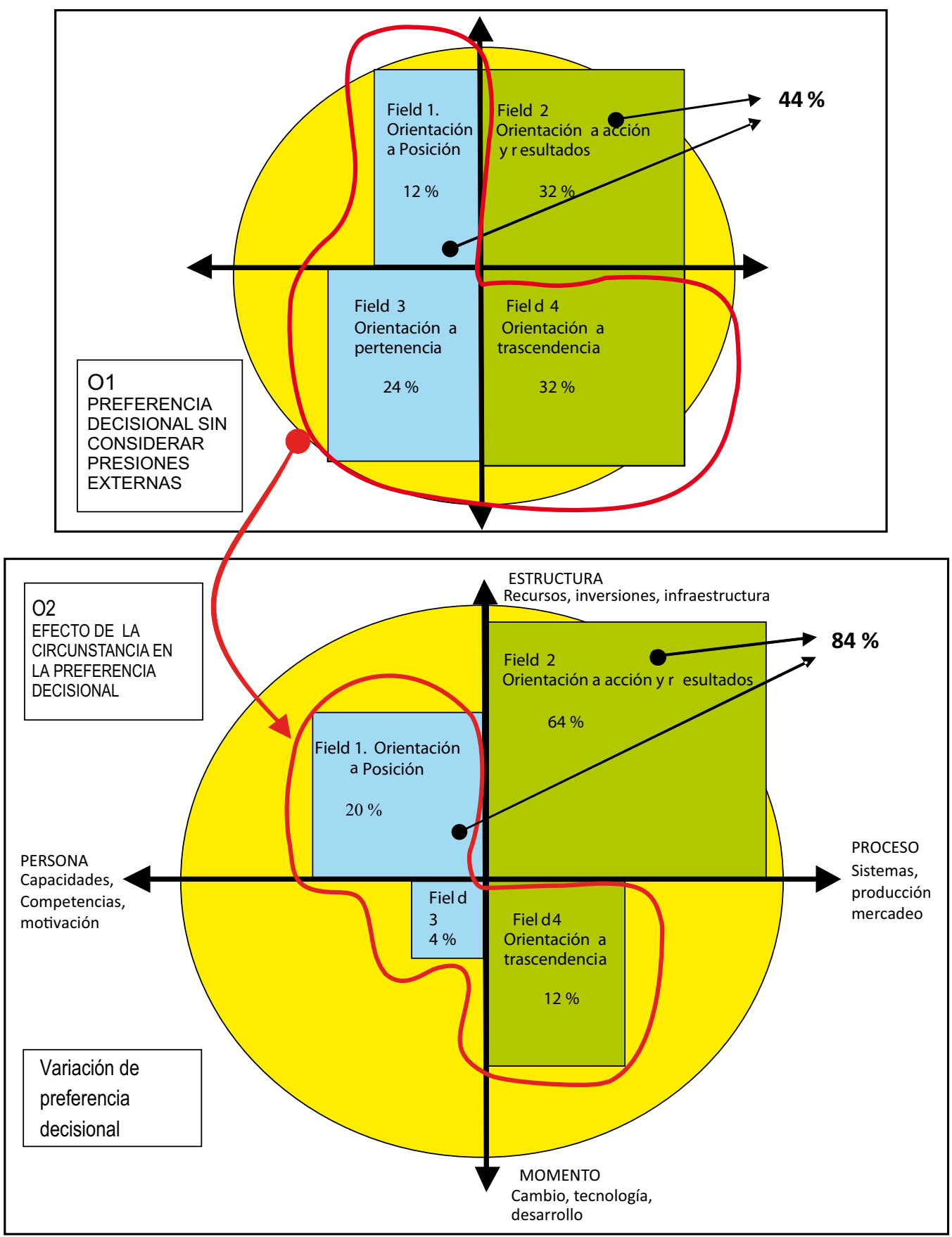

\section{CONCLUSIONES DEL ESTUDIO}

En la muestra, el análisis de diferencia de medias ha determinado que se rechaza la hipótesis básica y se acepta la hipótesis alternativa. Según la hipótesis alternativa, los decisores toman decisiones orientados por sus preferencias [7], pero que se ven afectadas y modificadas por la circunstancia y las presiones el entorno, bajo criterios de pragmatismo y utilitarismo.
El efecto principal de la circunstancia, en la orientación de las decisiones (Figura 8), sería:

- Los directivos se orientan a decisiones bajo criterios de utilitarismo, buscando resultados visibles, medidos en productividad y eficiencia. Esto es, las decisiones promedio pasan de cuadrantes de la izquierda hacia cuadrantes de la derecha. 
Figura 8. Efecto de la circunstancia en la orientación de la decisión - Resultados 1 y 2 del ga1

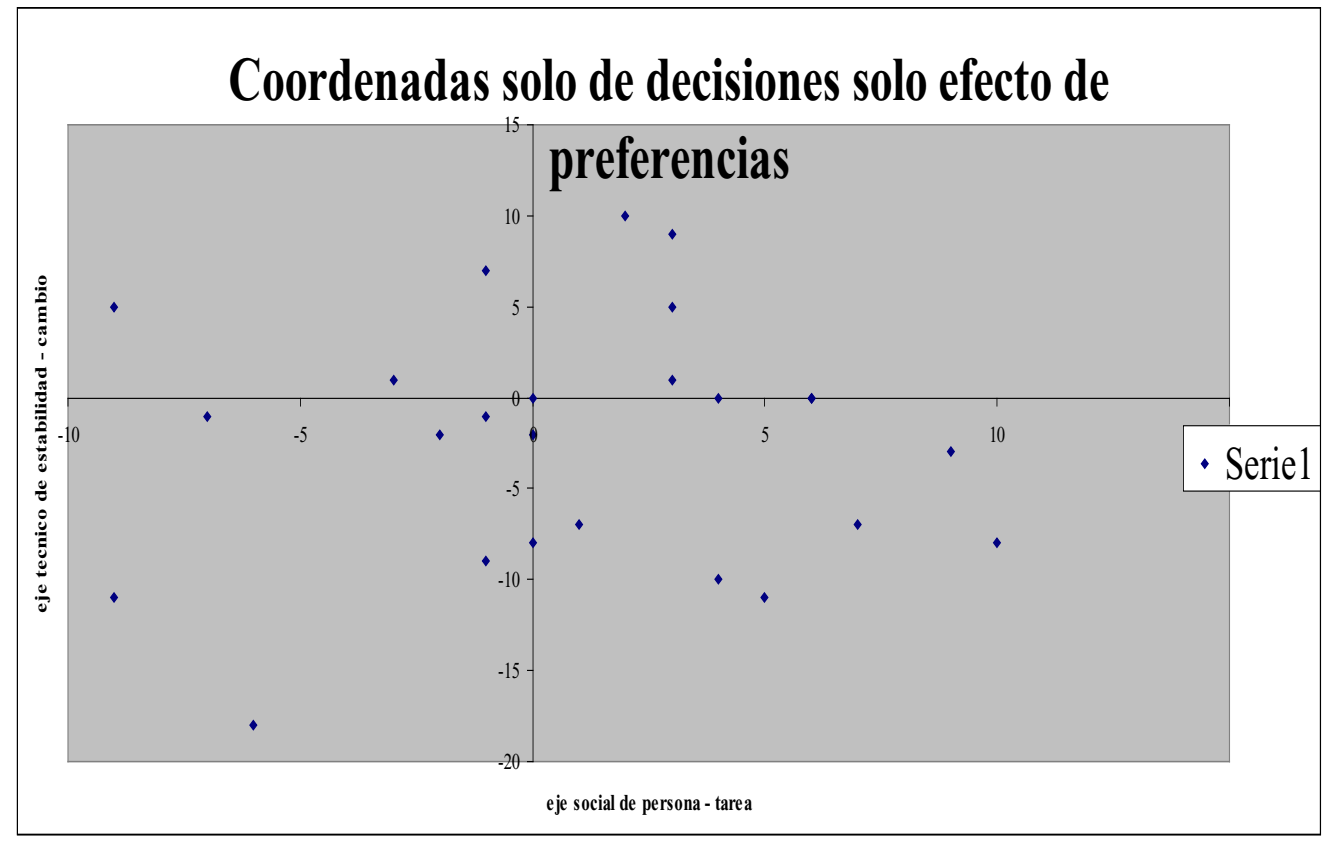

Fuente: Elaboración propia empleando SPSS y MS-Excel

- Los directivos se orientan a decidir bajo criterios de estabilidad, buscando reducir el riesgo de consecuencias no esperadas que afectarían su posición en la organización. Esto es, las decisiones promedio pasan de los cuadrantes inferiores hacia los cuadrantes superiores (menor cambio y mayor estabilidad).

- Los directivos asumen menor prioridad de las personas, cuando la situación del entorno se va tornando desfavorable.

- Los directivos asumen mayor prioridad de las tareas, cuando la situación del entorno se va tornando desfavorable.

- Si la situación problemática tiende a la inestabilidad, el desarrollo y el clima laboral (campo 3 y campo 4) pierden prioridad.

- No es posible determinar si, ante un entorno cambiante y agresivo en el largo plazo, la orientación decisional bajo criterios utilitarios, se consolida o, por el contrario, cambia si la circunstancia cambia.

- El decisor tiene una orientación hacia alguna dimensión de la empresa, para entender y resolver las situaciones problemáticas. Esta orientación básica es modificada por la circunstancia que afecta la percepción de la problemática, de modo que el entendimiento de los problemas se relaciona con la preferencia del decisor y la resolución de los problemas se ve influenciada por la circunstancia que rodea la situación problemática.

\section{REFERENCIAS BIBLIOGRÁFICAS}

[1] Acevedo, Adolfo (2010). Informe Técnico de proyecto "La circunstancia en la toma de decisiones y la resolución de problemas de empresa", investigación exploratoria y descriptiva FII UNMSM. Instituto de Investigación FII-UNMSM, Perú.

[2] Acevedo, Adolfo (2010). Informe Técnico de proyecto “"Las dimensiónes del modelo conceptual 4D para la resolución de problemas", investigación exploratoria y descriptiva FII UNMSM. Instituto de Investigación FII-UNMSM, Perú.

[3] Ackoff, Russell (2000). Recreación de las corporaciones. Un diseño organizacional para el siglo XXI. Ed. Oxford University Press. México.

[4] Báez, Juan (2009). Investigación cualitativa. 2. ${ }^{a}$ ed. ESIC Editorial. España.

[5] Blair y Whitson (1973). Elementos de Ingeniería de Sistemas Industriales. Editorial Prentice-Hall Internacional. España. 
[6] Checkland y Scholes (1994). La Metodología de los Sistemas Suaves de Acción. Noriega Editores. México.

[7] Duncan, Jack (2000). Las ideas y la práctica de la administración, Los principales desafíos en la era moderna, Oxford University Press, México.

[8] Fundación Drucker (1998). La organización del futuro. Ed. GRANICA, Argentina.

[9] Hernández, Roberto, Fernández, Carlos y Baptista, Lucio (2010). Metodología de la investigación. 5. ${ }^{\text {a }}$ ed. Ed. Mcgraw-Hill, Chile.

[10] Hodson, William (1996). Maynard Manual del Ingeniero Industrial. 4. ${ }^{\text {a }}$ ed. Ed. Mcgraw-Hill, México.

[11] INEI(2009). Boletín Especial N. ${ }^{\circ} 20$ "Perú: Estimaciones y Proyecciones de PEA Urbana y Rural, 2000- 2015.
[12] Kast y Rosenzweig (1968). Administración en las organizaciones, enfoque de sistemas y de contingencias. Ed. Mcgraw-Hill, México.

[13] Minztberg, Quinn y Voyer (1997). El proceso estratégico. Conceptos, contextos y casos. Prentice Hall. México.

[14] Riggs, James (2001). Sistemas de Producción. Planeación Análisis y Control. Limusa -Noriega Editores. México.

[15] Vaill, Peter (1967). "Industrial Engineering and Socio-Technical Systems", en Journal of Industrial Engineering, N. ${ }^{\circ}$ 9, vol. 16, USA.

[16] Zandin, Kjell (2005). Maynard Manual del Ingeniero Industrial. 5. ${ }^{\text {a }}$ ed. Ed. Mcgraw-Hill, México. 